\title{
Type II-P supernovae in the mid-infrared
}

\author{
Tamás Szalai and József Vinkó \\ Department of Optics and Quantum Electronics, University of Szeged, \\ Dóm tér 9., Szeged H-6720, Hungary; email: szaszi@titan.physx.u-szeged.hu
}

\begin{abstract}
We present detailed analysis of mid-infrared (MIR) data for 9 type II-P supernovae from the public Spitzer database. Spectral energy distributions (SEDs) from observed fluxes are fitted with simple models to get basic information about the dust as the presumed source of MIR radiation. We found two SNe, 2005ad and 2005af, which likely have newly-formed dust in their environment, while in the other seven cases the observed MIR flux may originate from pre-existing circumstellar or interstellar dust.
\end{abstract}

Keywords. supernova, circumstellar dust, infrared excess

\section{Unpublished supernova data in the Spitzer database}

Core-collapse supernovae (CC SNe), especially those of type II-plateau (II-P), are thought to be important contributors to cosmic dust production. The most obvious indicator of the presence of newly-formed and/or pre-existing dust is the time-dependent MIR excess coming from the environment of SNe. While in the past years several CC SNe were monitored by the Spitzer Space Telescope, there have been only a few of these objects analyzed and published up to now.

We studied the public Spitzer data of twelve type II-P (or peculiar II-P) SNe. In nine cases (SNe 2003J, 2003ie, 2004A, 2005ad, 2005af, 2006bp, 2006my, 2006ov, 2007oc), we could identify a mid-IR point source at the SN position, and carried out a complete analysis.

\section{Analysis of mid-IR data, model fitting of SEDs}

We computed simple aperture photometry on the post-BCD frames taken with Infrared Array Camera (IRAC), MIPS (Multiband Imaging Spectrometer) $24.0 \mu \mathrm{m}$ channel and IRS PUI (Infrared Spectrograph, peak-up imaging mode) with IRAF and MOPEX, taking into account all device-specific corrections. The available IRS spectra were processed using SPICE. MIR SEDs were calculated from observed fluxes. The continuum fluxes of reduced IRS spectra match well with the SED flux levels. Early-time MIR fluxes of SN 2005af, which are the only data already published elsewhere (Kotak et al. 2006), are also in good agreement with our values.

Assuming that the radiation is purely thermal, the main source of MIR flux is most likely warm dust. We fitted blackbodies (BBs) and analytic dust models to the observed, dereddened SED points to get information about physical properties and total amount of dust. Analytic models are based on Eq.1 in Meikle et al. (2007) assuming a homogeneous (constant-density) and uniform dust distribution within a sphere, using a power-law grain-size distribution. For most cases we used amorphous carbon (AC) grains (Colangeli et al. 1995), except for the cases of SNe 2005af and 2006my, where adequate solutions were possible only by applying a C-Si-PAH mixture (Weingartner \& Draine 2001). 

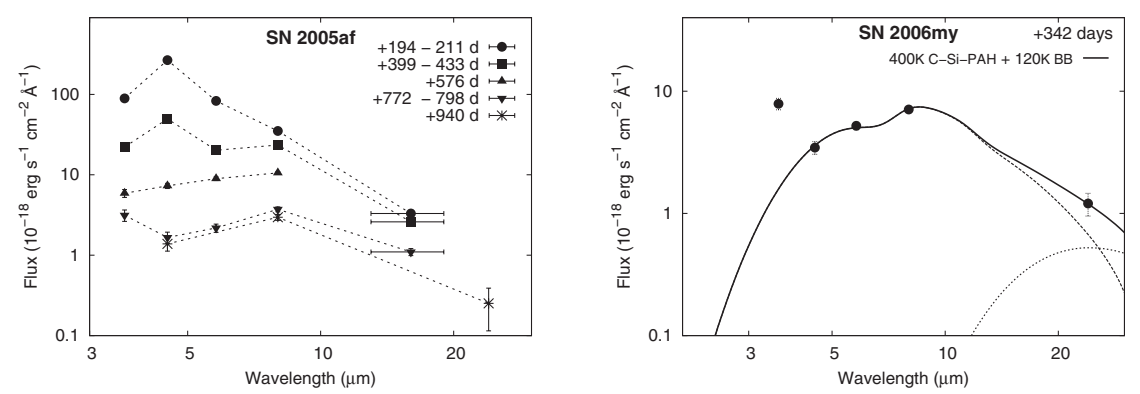

Figure 1. Left: Mid-IR SEDs of SN 2005af at different epochs. Right: An example of SED models: C-Si-PAH warm + cold BB components, SN 2006my (+342 days).

\section{Conclusions}

With the nine studied objects, we almost doubled the number of type II-P SNe having detailed MIR data based on Spitzer measurements. In two cases, SNe 2005ad and 2005af, we found cooling temperatures and decreasing luminosities of the warm component which are similar to the values found in other SNe that are thought to have newly-formed dust in their environment (e.g. SN 2004et - Kotak et al. 2009; SN 2004dj - Szalai et al. 2011, Meikle et al. 2011).

The calculated temperatures for the other SNe do not show strong temporal variation, while the derived luminosities as well as radii, are too high to be compatible with local dust. Also, the calculated dust masses in these cases are orders of magnitudes higher than the observed amount of dust around well-studied SNe listed above. The large radius of the warm component may suggest pre-existing dust in these cases, thereby making it unclear if there was new dust formed around these SNe.

Nevertheless, theoretical models predict orders of magnitude more newly-formed dust in CC SNe. Our conclusions support the previous observational results that warm new dust in the environment of SNe contributes only slightly to cosmic dust content. A more important contributor, as latest results suggest, may be the colder $(<50 \mathrm{~K})$ dust which could be found in older SN remnants (see e.g. Matsuura et al. 2011).

\section{Acknowledgments}

This work is supported by the Hungarian OTKA Grant K76816, by the European Union and co-funded by the European Social Fund through the TÁMOP 4.2.2/B-10/ 1-2010-0012 grant.

\section{References}

Colangeli, L., Mennella, V., Palumbo, P., et al. 1995, A\&AS, 113, 561

Kotak, R., Meikle, W. P. S., Pozzo, M., et al. 2006, ApJ (Letters), 651, L117

Kotak, R., Meikle, W. P. S., Farrah, D., et al. 2009, ApJ, 704, 306

Matsuura, M., Dwek, E., Meixner, M., et al. 2011, Science, 333, 1258

Meikle, W. P. S., Mattila, S., Pastorello, A., et al. 2007, ApJ, 665, 608

Meikle, W. P. S., Kotak, R., Farrah, D., et al. 2011, ApJ, 732, 109

Szalai, T., Vinkó, J., Balog, Z., et al. 2011, A\&̇A, 527, A61

Weingartner, J. C. \& Draine, B. T. 2001, ApJ, 548, 296 